\title{
单晶 $\alpha-\mathrm{Si}_{3} \mathbf{N}_{4}$ 纳米线宏量制备研究
}

$$
\text { 雷 超 }{ }^{1,2} \text {, 魏飞 }{ }^{2}
$$

(1. 广东省科学院 广东省材料与加工研究所, 广州 510650; 2. 清华大学 化工系, 绿色反应工程与工艺北京市重 点实验室, 北京 100084)

摘 要: 本研究提出了一种宏量制备单晶 $\alpha-\mathrm{Si}_{3} \mathrm{~N}_{4}$ 纳米线的方法。以造粒硅粉为原料, 通过在 $\mathrm{N}_{2}-\mathrm{H}_{2}$ 混合气氛中直接 氮化, 得到具有核壳结构的氮化产物 $\left(\mathrm{Si}_{3} \mathrm{~N}_{4}\right.$ 纳米线@多孔 $\mathrm{Si}_{3} \mathrm{~N}_{4}$ 微米粉体), 氮化产物经过破碎、研磨、分离后即可 获得 $\mathrm{Si}_{3} \mathrm{~N}_{4}$ 纳米线。检测结果表明, 制备的 $\mathrm{Si}_{3} \mathrm{~N}_{4}$ 纳米线直径为 $80 \sim 150 \mathrm{~nm}$, 长径比为 $20 \sim 50$, 其中纳米线含量 $>95 \mathrm{wt} \%, \alpha$ 相 $/ \beta$ 相比为 17.6, 收率为 3.1\%。进一步研究表明, 原料中微量 $\mathrm{Fe}$ 元素在还原气氛下具有催化作用, 纳 米线由典型的气一液-固(VLS)生长机制控制。实验中对原料硅粉造粒后再氮化具有三大优点: 数量级地增大了 $\mathrm{Si}_{3} \mathrm{~N}_{4}$ 纳米线生长空间; 纳米线生长分布集中, 有利于后续高效分离; 显著提高了氮化速率。

关 键 词: 氮化硅; 纳米线; 直接氮化; 造粒; VLS 机制

中图分类号: TB32 文献标识码: A

\section{Mass Production of $\alpha$-silicon Nitride Single-crystalline Nanowires}

\author{
LEI Chao ${ }^{1,2}$, WEI Fei ${ }^{2}$ \\ (1. Guangdong Institute of Materials and Processing, Guangdong Academy of Sciences, Guangzhou 510650, China; Beijing \\ Key Laboratory of Green Chemical Reaction Engineering and Technology, Department of Chemical Engineering, Tsinghua \\ University, Beijing 100084, China)
}

\begin{abstract}
Si powders in $\mathrm{N}_{2}-\mathrm{H}_{2}$ mixture gas. The nitridation product has the core-shell structure $\left(\mathrm{Si}_{3} \mathrm{~N}_{4}\right.$ nanowires @ porous $\mathrm{Si}_{3} \mathrm{~N}_{4}$ powders), where $\mathrm{Si}_{3} \mathrm{~N}_{4}$ nanowires can be effectively separated by crushing and grinding. The results show that the as-prepared $\alpha-\mathrm{Si}_{3} \mathrm{~N}_{4}$ nanowires are straight and uniform with diameters of $80-150 \mathrm{~nm}$, length to diameter ratios of 20-50, purity higher than $95 \mathrm{wt} \%$, and yield of 3.1\%. Further study indicates that growth of $\mathrm{Si}_{3} \mathrm{~N}_{4}$ nanowires is controlled by the Vapor-Liquid-Solid (VLS) mechanism, where the trace Fe elements serves as catalyst in the reduction atmosphere. In this study, The process of the raw silicom powder granulation after nitriding shows three advantages: 1) remarkably increasing growth space of nanowires; 2) leading to concentrated distribution of nanowire, tacilitating subsequent separation; 3 ) remarkably increasing the nitridation rate.
\end{abstract}

Key words: $\mathrm{Si}_{3} \mathrm{~N}_{4}$; nanowires; direct nitridation; granulation; VLS mechanism

收稿日期: 2018-08-31; 收到修改稿日期：2018-10-03

基金项目: 广东省科学院实施创新驱动发展能力建设专项(2018GDASCX-0964); 广东省科学院科研平台环境与能力建设专项 (2016GDASPT-0209，2016GDASPT-0321); 广东省科学院院属骨干科研机构创新能力建设专项(2017GDASCX0117, 2018GDASCX-0117); 广州市科技计划项目(ZWY201704003); 广东省材料与加工研究所创新能力建设项目 (2017A070701029); 广东省公益研究与能力建设专项(2017A070702019); 广东省省级科技计划项目(2017A050503004) GDAS' Project of Science and Technology Development (2018GDASCX-0964); GDAS' Project of Research Environment and Capacity Building (2016GDASPT-0209, 2016GDASPT-0321); Guangdong Academy of Sciences Project (2017GDASCX-0117, 2018GDASCX-0117); Guangzhou Science and Technology Planning Project (ZWY201704003); Guangdong Institute of Materials and Processing Innovation Capacity Building Project (2017A070701029); Project for Public Welfare Research and Capacity Building of Guangdong Province (2017A070702019); Science and Technology Planning Project of Guangdong Province (2017A050503004)

作者简介: 雷 超(1988-), 男, 博士. E-mail: chleijob@163.com 
氮化硅 $\left(\mathrm{Si}_{3} \mathrm{~N}_{4}\right)$ 是一种人工合成的高温结构材料, 同时也是新型功能材料, 具有化学稳定性好、电阻 率高、耐高温、抗热冲击、辐射硬度高、机械性能 好和光学性能优良等特性 ${ }^{[1-2]} 。 \mathrm{Si}_{3} \mathrm{~N}_{4}$ 纳米线是氮化 硅的一维纳米结构形式, 其不仅具有体相 $\mathrm{Si}_{3} \mathrm{~N}_{4}$ 材 料所拥有的各种优异性能, 作为一维纳米材料还具 备了许多新的特性。譬如: $\mathrm{Si}_{3} \mathrm{~N}_{4}$ 纳米线的抗弯强度 与弾性模型(达 $570 \mathrm{GPa}$ )远高于块体材料, 且具有极 好的柔韧性 ${ }^{[3]}$; 氮化硅纳米线是一维宽带隙半导体 材料 $(\sim 5.3 \mathrm{eV})$, 可以通过掺杂对其电学、光学性能 调控, 从而在纳米电子器件、光子器件领域有重要 的应用 ${ }^{[4]}$ 。

二十多年来, 研究人员相继开发了多种 $\mathrm{Si}_{3} \mathrm{~N}_{4}$ 纳米线制备方法, 如: 直接氮化法、碳热还原法、化 学气相沉积法、燃烧反应法、有机前驱体热解法、 溶剂热法和模板法等近十种 ${ }^{[5-15]}$ 。但由于投资和操 作费用过高、工业放大困难等问题, 绝大多数仍停 留在实验室制备阶段。据报道, 新西兰的 Nuenz 公 司于 2013 年率先在 $\mathrm{Si}_{3} \mathrm{~N}_{4}$ 纳米线商业化制备方面取 得突破, 其产物中 $\alpha-\mathrm{Si}_{3} \mathrm{~N}_{4}$ 含量大于 $95 \%$, 售价为 $\$ 10 / \mathrm{g}$ 。最近, 国内也开始有 $\mathrm{Si}_{3} \mathrm{~N}_{4}$ 纳米线商业化产 物问世, 其 $\alpha-\mathrm{Si}_{3} \mathrm{~N}_{4}$ 含量约为 $80 \sim 85 \%$, 售价在 $¥ 40 \sim 50 / \mathrm{g}$ 。总的来说, 国内外 $\mathrm{Si}_{3} \mathrm{~N}_{4}$ 纳米线材料仍 处于产业发展早期, 存在价格高、难以批量化生产 的问题, 无法满足日益增长的市场需求。因此, 开发 一种低成本、批量化的 $\mathrm{Si}_{3} \mathrm{~N}_{4}$ 纳米线制备技术对其 进一步发展与推广应用有重要意义。

针对此问题, 本研究提出一种直接氮化法制备 $\mathrm{Si}_{3} \mathrm{~N}_{4}$ 纳米线的方法, 即对细硅粉进行简单的造粒 预处理, 使得后续氮化过程中 $\mathrm{Si}_{3} \mathrm{~N}_{4}$ 纳米线生长在 造粒粉外表, 这样经过简单研磨破碎即可完成所得 $\mathrm{Si}_{3} \mathrm{~N}_{4}$ 纳米线与 $\mathrm{Si}_{3} \mathrm{~N}_{4}$ 粉体的分离。本方法的优点在 于: 操作简单, 易于批量化, 无需催化剂与后续纯化 除杂, 产物纯度高、易分离, 且可同时获得高品质 $\mathrm{Si}_{3} \mathrm{~N}_{4}$ 纳米线与 $\mathrm{Si}_{3} \mathrm{~N}_{4}$ 微米粉体。

\section{1 实验方法}

\section{1 材料制备}

实验原料为 $0.5 \sim 5.0 \mu \mathrm{m}$ 的不规则 $\mathrm{Si}$ 粉(图 1(a)), 纯度 $>99.95 \mathrm{wt} \%$ (购于徐州凌云硅业有限公司)。首 先, 将硅粉与 $1 \mathrm{wt} \%$ 的淀粉水溶液按质量比 $1: 1$ 混合 后, 摚拌升温至 $80{ }^{\circ} \mathrm{C}$ 并恒温 $2 \mathrm{~h}$, 将所得悬浊液喷 雾造粒得到尺寸为 50 200 $\mu \mathrm{m}$ 的多孔造粒 $\mathrm{Si}$ 粉球 (图 1(b))。
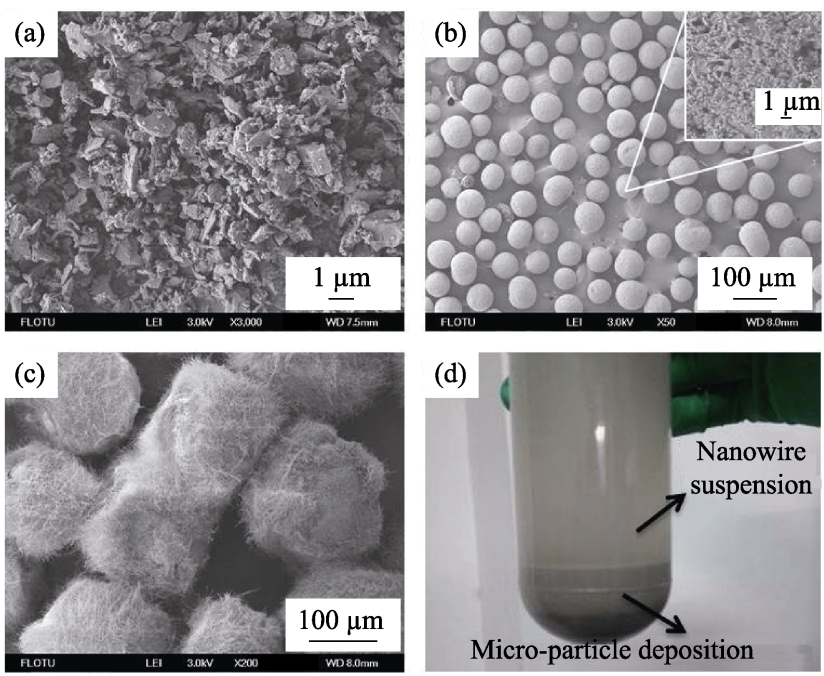

图 1 不同样品的 SEM 照片和实物照片

Fig. 1 SEM images and photograph of different samples (a) SEM image of Si raw materials; (b) SEM images of Granulation Si powders; (c) SEM image of granulation Si powders after $3 \mathrm{~h}$ nitridation; (d) Photograph of $\mathrm{Si}_{3} \mathrm{~N}_{4} /$ alcohol mixture after stratification

其次，在管式气氛炉中进行氮化反应。将 $10.0 \mathrm{~g}$ 造粒硅粉球置于刚玉瓷舟中, 在 $0.2 \mathrm{~L} / \mathrm{min}$ 的 $\mathrm{Ar}$ 保 护气氛下 $90 \mathrm{~min}$ 快速升温至 $1350{ }^{\circ} \mathrm{C}$, 之后在 $0.2 \mathrm{~L} / \mathrm{min}$ 的 $90 \mathrm{vol} \% \mathrm{~N}_{2} \sim 10 \mathrm{vol} \% \mathrm{H}_{2}$ 混合气下氮化并 保温 $3 \mathrm{~h}$, 反应结束后在 $\mathrm{Ar}$ 保护气氛下随炉冷却至 室温。

如图 1(c)所示, 氮化后球状颗粒表面被大量纳 米线包覆，包覆层厚度为 $10 \sim 50 \mu \mathrm{m}$ 。由于不同颗粒 外表层纳米线相互缠绕，使得产物为具有一定强度 的块状物。将所得块状氮化硅产物破碎、研磨呈单 分散颗粒状粉体。将研磨后的氮化硅粉体与乙醇混 合、摚拌, 由于 $\mathrm{Si}_{3} \mathrm{~N}_{4}$ 纳米线与 $\mathrm{Si}_{3} \mathrm{~N}_{4}$ 亚微米粉体的 沉降速度差异较大, 静置 5 10 min 后, 混合液将出 现分层(图 1(d)), 取混合液中的上层悬浊液进行离 心分离, 将所得白色固体物质干燥后即得 $\mathrm{Si}_{3} \mathrm{~N}_{4}$ 纳 米线产物。将混合液底部沉积物干燥后即得到 50 $200 \mu \mathrm{m}$ 的 $\mathrm{Si}_{3} \mathrm{~N}_{4}$ 亚微米粉体副产物。

\section{2 表征方法}

利用 JSM 7401F 型扫描电子显微镜(Scanning Electron Microscope, SEM)和 JEM 2010 型透射电子 显微镜(Transmission Electron Microscope, TEM)观 测样品的结构和形貌。产物氧含量由氮氧氢分析仪 检测 (TC-306, 上海宝英光电科技有限公司), 金属 杂质含量由电感耦合等离子体发射光谱仪(ICPAES)检测, 碳含量由碳硫分析仪检测。

用 Bruker D8 型 X 射线衍射仪(XRD)对样品的 结晶状态进行检测, 并基于 XRD 图谱对硅的转化 率、 $\alpha$ 相和 $\beta$ 相做定量分析 ${ }^{[16]}$ 。在计算三个相的质 
量分数时, 硅采用的是(111)峰的综合强度, $\alpha-\mathrm{Si}_{3} \mathrm{~N}_{4}$ 采用的是(102)、(210)、(201)峰的综合强度, 而 $\beta-\mathrm{Si}_{3} \mathrm{~N}_{4}$ 采用的是(101)、(210)峰的综合强度, 计算公式为:

$$
\begin{gathered}
\frac{I_{\alpha}(102)+I_{\alpha}(210)}{I_{\beta}(101)+I_{\beta}(210)}=0.647 \frac{W_{\alpha}}{W_{\beta}} \\
\frac{I_{\mathrm{si}}(11)}{I_{\alpha}(201)}=5.53 \frac{W_{\mathrm{si}}}{W_{\alpha}} \\
W_{\alpha}+W_{\beta}+W_{\mathrm{si}}=1
\end{gathered}
$$

式中, $I_{\delta}(h k l)$ 为 $\delta$ 相的 $h k l$ 衍射的综合强度; $W_{\delta}$ 为混 合物中 $\delta$ 相的质量分数。

由此可计算硅的总转化率 $X$ 为:

$$
X=\frac{1-W_{\mathrm{si}}}{1+0.665 W_{\mathrm{si}}}
$$

$\alpha$ 相、 $\beta$ 相的转化率 $X_{\alpha} 、 X_{\beta}$ 分别为:

$$
\begin{aligned}
& X_{\alpha}=\frac{1-W_{\mathrm{si}}}{1+0.665 W_{\mathrm{si}}} \times \frac{W_{\alpha}}{W_{\alpha}+W_{\beta}} \\
& X_{\beta}=\frac{1-W_{\mathrm{si}}}{1+0.665 W_{\mathrm{si}}} \times \frac{W_{\beta}}{W_{\alpha}+W_{\beta}}
\end{aligned}
$$

\section{2 结果与讨论}

\section{1 表征结果}

图 2 为制备的 $\mathrm{Si}_{3} \mathrm{~N}_{4}$ 纳米线、 $\mathrm{Si}_{3} \mathrm{~N}_{4}$ 微米粉体与 $\mathrm{Si}$ 粉原料的 XRD 图谱。分析结果表明, $\mathrm{Si}_{3} \mathrm{~N}_{4}$ 纳米 线产物氮化率为 $98.9 \%$, 以 $\alpha$ 相为主 $(\alpha$ 相 $/ \beta$ 相 $=17.6)$, 杂质为少量未转化的硅单质。所得 $\mathrm{Si}_{3} \mathrm{~N}_{4}$ 微米粉体 的氮化率为 $91.7 \%$, 其中 $\alpha$ 相 $/ \beta$ 相 $=15.2$, 杂质主要 为少量未转化的硅单质。

表 1 为 $\mathrm{Si}_{3} \mathrm{~N}_{4}$ 纳米线、 $\mathrm{Si}_{3} \mathrm{~N}_{4}$ 微米粉体与 $\mathrm{Si}$ 粉原 料的杂质组成分析。结果表明, 原料 $\mathrm{Si}$ 纯度较高, 主要金属杂质为 $\mathrm{Fe} 、 \mathrm{Ca} 、 \mathrm{Al}$ 三种元素。所制备的 $\mathrm{Si}_{3} \mathrm{~N}_{4}$ 纳米线与 $\mathrm{Si}_{3} \mathrm{~N}_{4}$ 微米粉体中, $\mathrm{Fe} 、 \mathrm{Ca}$ 元素含量 与原料中含量相当, 仅 $\mathrm{Al}$ 含量轻微升高, 这主要是

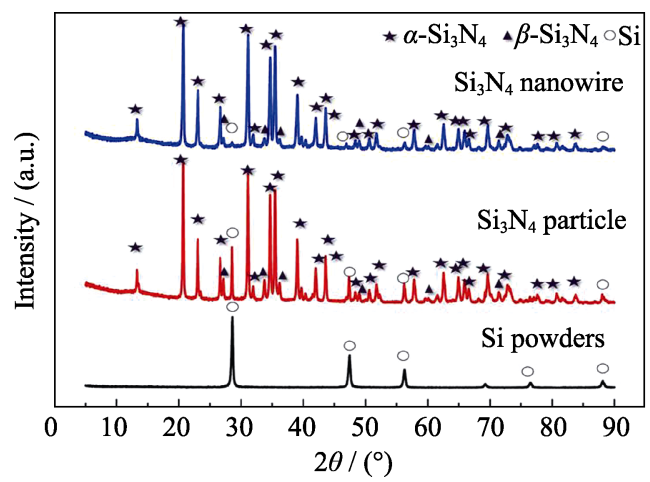

图 $2 \mathrm{Si}$ 粉原料、 $\mathrm{Si}_{3} \mathrm{~N}_{4}$ 粉体、 $\mathrm{Si}_{3} \mathrm{~N}_{4}$ 纳米线的 $\mathrm{XRD}$ 图谱 Fig. 2 XRD patterns of Si raw materials, $\mathrm{Si}_{3} \mathrm{~N}_{4}$ powders, $\mathrm{Si}_{3} \mathrm{~N}_{4}$ nanowires
表 1 杂质元素的分析结果

Table 1 Impurity elements analysis results

\begin{tabular}{cccccc}
\hline Element $/$ wt $\%$ & $\mathrm{Fe}$ & $\mathrm{Ca}$ & $\mathrm{Al}$ & $\mathrm{O}$ & $\mathrm{C}$ \\
\hline Si raw materials & 0.008 & 0.013 & 0.012 & 0.14 & - \\
$\mathrm{Si}_{3} \mathrm{~N}_{4}$ nanowires & 0.011 & 0.007 & 0.031 & 1.56 & - \\
$\mathrm{Si}_{3} \mathrm{~N}_{4}$ particles & 0.006 & 0.014 & 0.049 & 1.07 & - \\
\hline
\end{tabular}

由于刚玉管与刚玉瓷舟在高温下存在一定程度的挥 发导致的。非金属杂质方面, 由于体系存在微量氧, $\mathrm{Si}_{3} \mathrm{~N}_{4}$ 纳米线与 $\mathrm{Si}_{3} \mathrm{~N}_{4}$ 微米粉体的氧含量分别达到 $1.56 \mathrm{wt} \% 、 1.07 \mathrm{wt} \%$, 均高于原料。

值得注意的是, 本实验中添加淀粉的作用是造 粒，而不考虑其他副作用，主要是基于以下两点分析：

i) 对造粒硅粉氮化前后的碳含量检测分别为 $0.082 \mathrm{wt} \% \sim 0.097 \mathrm{wt} \%$ 与 $0.025 \mathrm{wt} \% \sim 0.039 \mathrm{wt} \%$ ，显著 低于淀粉的加入量 $(1 \mathrm{wt} \%)$, 这主要是由于：淀粉在 高温下发生热分解，大部分以 $\mathrm{CO}_{2}$ 和 $\mathrm{H}_{2} \mathrm{O}$ 形式挥发， 当体系温度高于 $1000{ }^{\circ} \mathrm{C}$ 时, 残留碳灰分的重量低 于 $1 / 10^{[17]}$, 故此部分含碳灰分在产物中的含量将小 于 $0.1 \mathrm{wt} \%$; 残留的碳灰分在高温 $\mathrm{N}_{2}-\mathrm{H}_{2}$ 混合气氛下, 可与 $\mathrm{H}_{2}$ 反应生成 $\mathrm{CH}_{4}$, 被进一步消耗 ${ }^{[18]}$, 因此残留 在产物中单质碳的含量会进一步降低。由于氮化过 程中残留的碳灰分对本体系不存在催化作用, 且含 量极低, 其对氮化反应在热力学与动力学上的影响 可以忽略不计。

ii) 所使用淀粉符合马铃薯淀粉标准 GB/T8884, 其中主要铅、砷等金属杂质总含量小于 $10^{-6}$, 非金 属硫元素含量小于 $10^{-5}$ 。因此硅粉球由淀粉引入的 杂质总含量极少(小于 $10^{-7}$ ), 而目前也尚未有以上 元素对直接氮化反应影响的相关报道, 故其对氮化 反应在热力学与动力学上的影响可忽略不计。

图 3 为 $\mathrm{Si}_{3} \mathrm{~N}_{4}$ 纳米线产物形貌, 从图可知, 纳米 线中夹杂有少量 $0.5 \sim 2.0 \mu \mathrm{m}$ 的亚微米颗粒, 进一步 通过统计多张 SEM 照片中 $\mathrm{Si}_{3} \mathrm{~N}_{4}$ 纳米线与颗粒状 $\mathrm{Si}_{3} \mathrm{~N}_{4}$ 的面积, 计算得到产物中纳米线的含量大于 $95 \%$ 。单根纳米线长度为 $1 \sim 5 \mu \mathrm{m}$, 直径 $80 \sim 150 \mathrm{~nm}$, 长径比为 20 50。对比图 1(c) 可知, 图 3(a)中纳米线 明显变短, 这主要是由于后期研磨破碎所致。高倍 率透射电镜观察结果(图 3(c) (d))表明, 纳米线无固 定生长方向。分区衍射结果表明，所制备的纳米线 均为单晶结构。此外，纳米线表面光滑，部分纳米线 外层有厚度 $0.5 \sim 1.0 \mathrm{~nm}$ 的无定型层, 这种无定型包 覆层在氮化硅纳米线的制备中较为常见, 一般认为 主要是体系中微量的氧导致生成 $\mathrm{SiO}_{2}$ 所致 ${ }^{[19-20]}$ 。由 于含量较少且为无定型状态, 在 XRD 测试结果中 未能显示。对纳米线进行 EDS 元素分析结果也表明, 纳米线表面有约 3at\%的 O 元素(表 2)。 

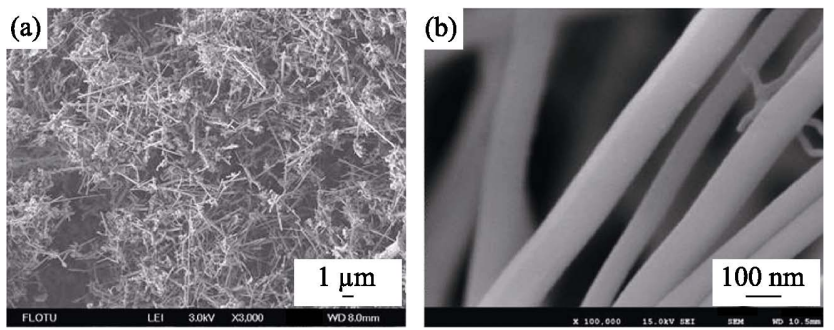

(c)
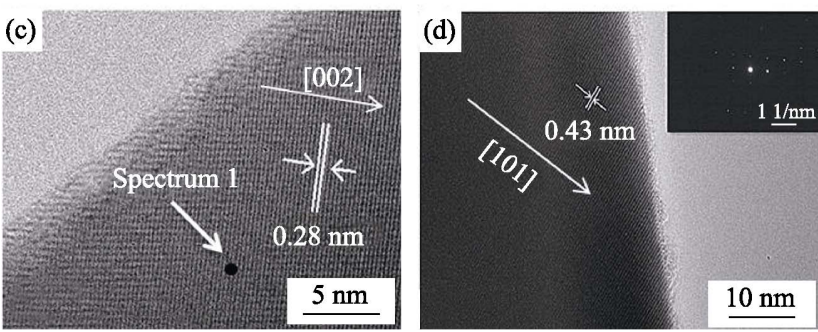

图 $3 \mathrm{Si}_{3} \mathrm{~N}_{4}$ 纳米线的形貌照片

Fig. 3 Morphologies of $\mathrm{Si}_{3} \mathrm{~N}_{4}$ nanowires

(a-b) SEM images; (c) TEM imag; (d) SAED pattern

表 2 纳米线表面的 EDS 元素分析结果

Table 2 Elemental analysis results of $\mathrm{Si}_{3} \mathrm{~N}_{4}$ nanowires

\begin{tabular}{ccccc}
\hline \multirow{2}{*}{ Spectrum } & \multicolumn{4}{c}{ Element/at\% } \\
\cline { 2 - 5 } & $\mathrm{Si}$ & $\mathrm{N}$ & $\mathrm{O}$ & $\mathrm{Fe}$ \\
\hline 1 & 41.42 & 55.43 & 3.16 & 0 \\
2 & 49.17 & 46.22 & 4.17 & 0.45 \\
\hline
\end{tabular}

图 4 为 $\mathrm{Si}_{3} \mathrm{~N}_{4}$ 微米粉体形貌照片。对比图 1(b) (c) 与图 4(a) (b)可知, 造粒硅粉球氮化后生成的 $\mathrm{Si}_{3} \mathrm{~N}_{4}$ 微米粉体仍保持了初始的多孔球形特征, 并且由于 高温氮化导致内部亚微米粉体之间存在一定程度的 烧结, $\mathrm{Si}_{3} \mathrm{~N}_{4}$ 微米粉体具有较高的强度, 经过破碎研 磨后仅发生轻度破碎, 其平均粒径从初始的 $147 \mu \mathrm{m}$ 下降至 $93 \mu \mathrm{m}$ 。研磨后的 $\mathrm{Si}_{3} \mathrm{~N}_{4}$ 粉体外表层的纳米线 层脱落较完全, 颗粒表面仅粘附有短纳米线 $(0.1 \sim 3.0 \mu \mathrm{m})$, 粉体内部也有少量纳米线, 但由于生 长空间限制, 数量少且短 $(<1 \mu \mathrm{m})$ 。

\section{2 生长机制}

一般来说，直接氮化法制备 $\mathrm{Si}_{3} \mathrm{~N}_{4}$ 纳米线的生
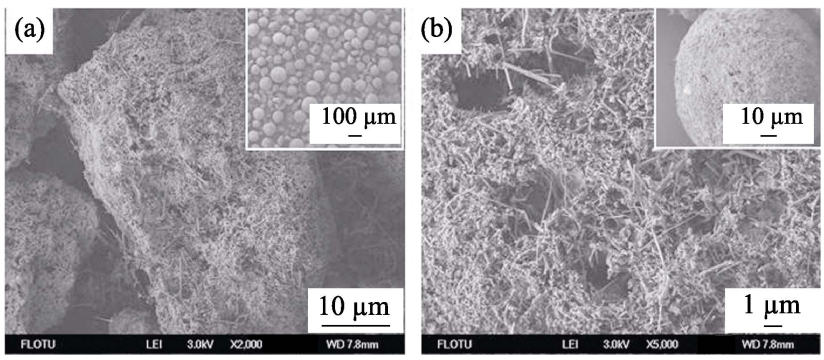

图 4 不同倍率下 $\mathrm{Si}_{3} \mathrm{~N}_{4}$ 微米粉体的形貌照片

Fig. 4 Morphologies under different magnifications for $\mathrm{Si}_{3} \mathrm{~N}_{4}$ particles

(a) Broken particle; (b) Unbroken particle

长机制主要有两种：气-液-固(VLS)机制和气一固 $(V S)$ 机制 ${ }^{[21]}$ 。VLS 机制的典型过程为: 高温下气相 中 $\mathrm{N}_{2} 、 \mathrm{Si}$ 蒸气与作为催化剂的熔融态金属颗粒形成 固溶体, 达到过饱和后 $\mathrm{Si}_{3} \mathrm{~N}_{4}$ 从催化剂中析出形核, 由于气相分子不断地进入到液态金属中溶解、析出, 从而使晶体得以生长形成一维纳米材料 ${ }^{[22]}$ 。VS 生 长机制无需催化剂，气相原料经输运传至基片表面 沉积下来、生长, 形成一维纳米材料。两种机制可 以通过纳米线顶端(或底部)是否有由含有金属相的 球形圆头形成加以区分判定。

为了探究本实验中 $\mathrm{Si}_{3} \mathrm{~N}_{4}$ 纳米线的生长机制, 对 $\mathrm{Si}_{3} \mathrm{~N}_{4}$ 纳米线生长初期 $(15 \mathrm{~min}$ )的形貌进行了考 察。如图 5(a) (b)所示, 球形硅粉氮化 $15 \mathrm{~min}$ 后表 面开始长出 $\mathrm{Si}_{3} \mathrm{~N}_{4}$ 纳米线, 此时大部分 $\mathrm{Si}_{3} \mathrm{~N}_{4}$ 纳米线 顶端有球形圆头。EDS 分析结果表明(表 2), 纳米线 顶端圆球含量约 $0.45 \mathrm{wt} \%$ 的 $\mathrm{Fe}$ 元素(约为 $\mathrm{Si}$ 粉原料 含量的 60 倍), 而纳米线体相没有检测到 $\mathrm{Fe}$ 元素。因 此，可推断本实验中纳米线的生成机制为 VLS 机制。

由于本实验未向体系中添加 $\mathrm{Fe}$ 元素, 故推断纳 米线顶端的 $\mathrm{Fe}$ 来自于 $\mathrm{Si}$ 粉原料体相。为此, 进一 步考察了硅粉球在纯 $\mathrm{N}_{2}$ 气氛下的氮化行为。如 图 5(c)所示, 发现当氮化气氛中不加入氢气时, 硅 粉球在 $1350{ }^{\circ} \mathrm{C}$ 下氮化 $3 \mathrm{~h}$ 后, 表面仅有少量由 VS 机制生成的短 $\mathrm{Si}_{3} \mathrm{~N}_{4}$ 纳米线 $(1 \sim 2 \mu \mathrm{m})$, 而无法形成如
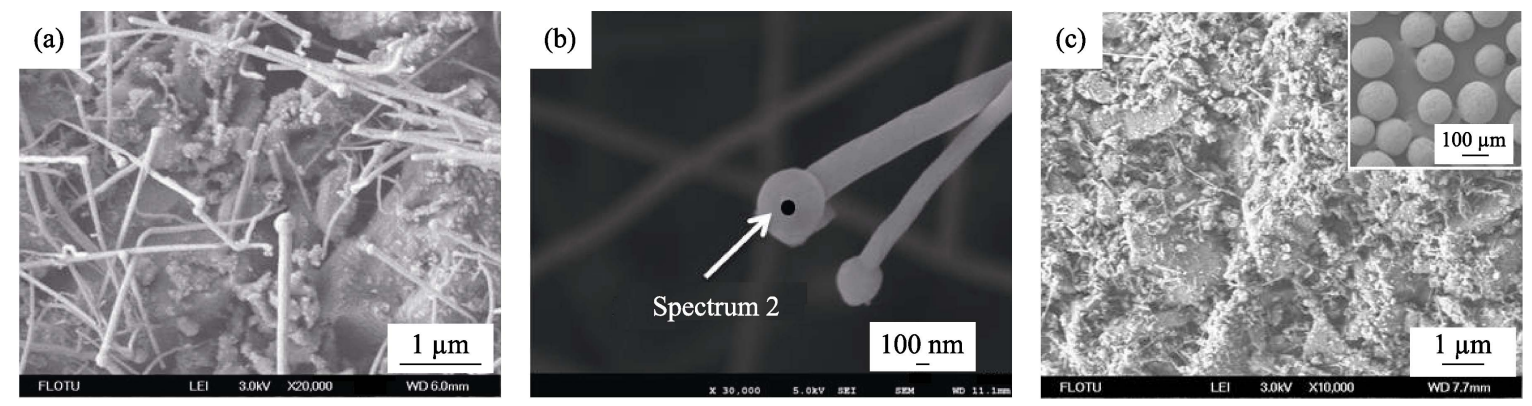

图 5 硅粉球表面纳米线的形貌照片

Fig. 5 Morphologies of nanowires on the granulation Si powder surface (a-b) 15 min nitridation in the $90 \mathrm{vol} \% \mathrm{~N}_{2}-10 \mathrm{vol} \% \mathrm{H}_{2}$ mixture gas; (c) $3 \mathrm{~h}$ nitridation in $\mathrm{N}_{2}$ 
图 1(c)中由大量长 $\mathrm{Si}_{3} \mathrm{~N}_{4}$ 纳米线形成的包裹层。这就 佐证了本实验中 $\mathrm{Si}_{3} \mathrm{~N}_{4}$ 纳米线为 VLS 生长机制, 即 高温氮化过程中, 硅粉中的 $\mathrm{Fe}$ 在还原气氛下与 $\mathrm{Si}$ 、 $\mathrm{N}$ 元素形成合金液滴，作为 $\mathrm{Si}_{3} \mathrm{~N}_{4}$ 纳米线的生长催化 剂。而在纯氮气中, 体系中含有 $10^{-6}$ 级的氧气, 以 及硅粉自身外表层的氧化包覆层难以去除, 无法形 成 VLS 机制所需的合金液滴相，故没有纳米线大量 生成。

以上研究表明, 本研究中在不使用催化剂的条 件下, 对硅粉进行喷雾造粒后氮化, 制备了具有核 壳结构的 $\mathrm{Si}_{3} \mathrm{~N}_{4}$ 纳米线@ $\mathrm{Si}_{3} \mathrm{~N}_{4}$ 微米粉体。相比于 $\mathrm{Si}$ 粉直接氮化，对其预造粒的优点在于:

1) 纳米线生长空间大。图 6(a) 为未造粒硅粉 在 $1350{ }^{\circ} \mathrm{C} 、 90 \mathrm{vol} \% \mathrm{~N}_{2}+10 \mathrm{vol} \% \mathrm{H}_{2}$ 混合气氛下氮化 $3 \mathrm{~h}$ 后的形貌照片, 从中可以看出, 未造粒硅粉氮化 后的形貌与造粒硅粉内部颗粒近似(图 6(b)), 由于 颗粒间的间隙距离小 $(0.5 \sim 2.0 \mu \mathrm{m})$, 氮化后颗粒表面 生长的纳米线短 $(0.5 \sim 2.0 \mu \mathrm{m})$, 且较为稀疏。相比而 言, 由于造粒硅粉球之间的间隙距离大 $(10 \sim 50 \mu \mathrm{m})$, 氮化后造粒球外部间隙被大量长而密集的纳米线所 填满(图 1(c)), 这样便数量级地扩大了纳米线生长 空间，进而提高了纳米线的产率。本实验中 $\mathrm{Si}_{3} \mathrm{~N}_{4}$ 纳米线产率约为 $3.1 \%$ 。

2) 纳米线集中分布于硅粉球外表, 易分离。从 图 6(a)可知, 未造粒粉直接氮化后, 颗粒与短纳米 线间相互缠绕与烧结, 目前尚无有效的手段将纳米 线与颗粒分离。而造粒硅粉氮化后具有 $\mathrm{Si}_{3} \mathrm{~N}_{4}$ 纳米 线@ $\mathrm{Si}_{3} \mathrm{~N}_{4}$ 微米粉体的核壳结构。内部多孔 $\mathrm{Si}_{3} \mathrm{~N}_{4}$ 微 米粉体由于高温氮化引发颗粒烧结, 其强度极高, 在后续研磨中仅会轻度破碎, 但其粒径一般仍大于 $50 \mu \mathrm{m}$ 。外层集中生长 $\mathrm{Si}_{3} \mathrm{~N}_{4}$ 纳米线不会发生烧结, 仅仅处于相互缠绕状态, 强度较低, 在破碎、研磨过 程中易于内部 $\mathrm{Si}_{3} \mathrm{~N}_{4}$ 微米粉体分离。

3) 氮化速率高。以往研究报道表明, 亚微米硅
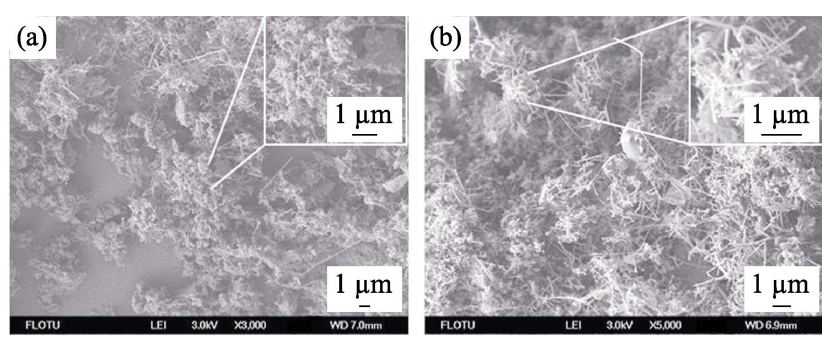

图 6 不同硅在 $1350{ }^{\circ} \mathrm{C}, 90 \mathrm{vol} \% \mathrm{~N}_{2}+10 \mathrm{vol} \% \mathrm{H}_{2}$ 混合气氛下 氮化 $3 \mathrm{~h}$ 后的 SEM 照片

Fig. 6 SEM images of different Si powders after $3 \mathrm{~h}$ nitridation in the $90 \mathrm{vol} \% \mathrm{~N}_{2}+10 \mathrm{vol} \% \mathrm{H}_{2}$ mixture gas (a) Raw Si powders; (b) Inside of the granulation Si powders

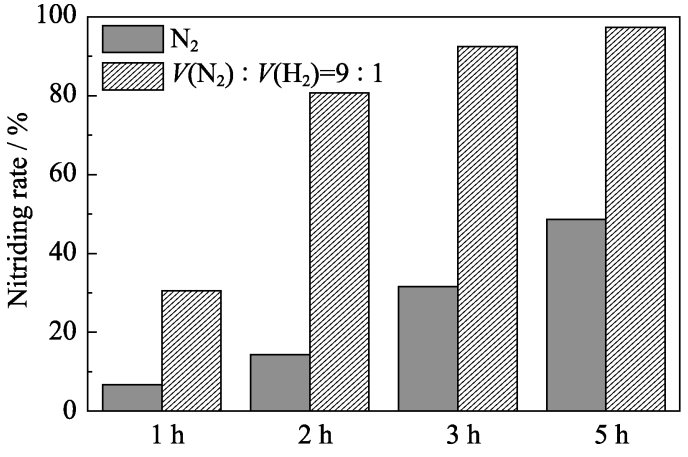

图 7 不同气氛下, 造粒硅粉球的氮化速率

Fig. 7 Nitridation rate of granulation Si powders at different atmospheres

粉一般需在 $1300 \sim 1350{ }^{\circ} \mathrm{C}$ 氮化 $10 \sim 20 \mathrm{~h}$ 后, 氮化率 才能超过 $90 \%{ }^{[23]}$, 而本实验仅需 $3 \mathrm{~h}$ 。这主要是由于: i) $\mathrm{Si}_{3} \mathrm{~N}_{4}$ 纳米线的生成提高了整体反应速率。如图 5(c) 与图 7 所示, 当硅粉球在 $\mathrm{N}_{2}$ 气氛下氮化时, $\mathrm{Si}_{3} \mathrm{~N}_{4}$ 产 物致密覆盖在硅粉颗粒外表面, 使得反应阻力不断 增大，反应 $3 \mathrm{~h}$ 后氮化率仅为 $31.67 \%$ 。当硅粉球在 $90 \mathrm{vol} \% \mathrm{~N}_{2}+10 \mathrm{vol} \% \mathrm{H}_{2}$ 气氛下氮化时，早期氮化硅 产物主要以 $\mathrm{Si}_{3} \mathrm{~N}_{4}$ 纳米线生长在颗粒外表，而不是 致密覆盖在反应物外层, 减轻了反应阻力, 硅粉球 氮化 $1 \mathrm{~h}$ 后氮化硅即达到 $30.5 \%, 5 \mathrm{~h}$ 后已达 $97.3 \%$ 。 ii)氮化过程中一般将亚微米硅粉直接堆积进行反应, 由于气体扩散阻力大，产生内部物料氮化率往往低 于外层物料，出现“流芯”现象。 $1350{ }^{\circ} \mathrm{C} 、 90 \mathrm{vol} \%$ $\mathrm{N}_{2}+10 \mathrm{vol} \% \mathrm{H}_{2}$ 混合气氛下造粒粉氮化 $3 \mathrm{~h}$ 后的氮化 率为 $68.3 \%$; 而相同条件下，硅粉经过造粒后氮化 $3 \mathrm{~h}$, 其氮化率达 $92.5 \%$ 。这是因为造粒颗粒的间隙 距离较大，有利于 $\mathrm{N}_{2}$ 向物料层内部扩散，使得反应 速率更快，整体反应更均一。

综上所述，本实验所提出的 $\mathrm{Si}_{3} \mathrm{~N}_{4}$ 纳米线制备 方法，优点如下: 1)无需添加催化剂、方法简单、氮 化速率快、可批量化生产。本方法基于已实现工业 化的直接氮化法，仅额外添加了易于规模化操作的 喷雾造粒与研磨分离两道工序; 2)产物品质好，且 无需后续纯化除杂。所制备的 $\alpha-\mathrm{Si}_{3} \mathrm{~N}_{4}$ 纳米线产物中 纳米线含量大于 $95 \%, \alpha$ 相 $/ \beta$ 相比为 $17.6 ; 3)$ 纳米线 收率高、易分离。 $\mathrm{Si}_{3} \mathrm{~N}_{4}$ 纳米线仅需研磨破碎即可实 现与 $\mathrm{Si}_{3} \mathrm{~N}_{4}$ 粉体的高效分离, 收率可达 $3.1 \%$ 。

\section{3 结论}

本研究提出了一种以造粒硅粉球为原料, 通过 高温直接氮化，宏量制备单晶 $\alpha-\mathrm{Si}_{3} \mathrm{~N}_{4}$ 纳米线的方 法。研究结果表明: 原料硅粉造粒后再氮化具有三大 优点: 1)数量级地增大 $\mathrm{Si}_{3} \mathrm{~N}_{4}$ 纳米线生长空间; 2)纳米 
线生长分布集中，有利于后续高效分离; 3)显著提高 氮化速率。所制备的 $\mathrm{Si}_{3} \mathrm{~N}_{4}$ 纳米线直径为 $80 \sim 150 \mathrm{~nm}$, 其长径比为 $20 \sim 50$, 纳米线含量大于 $95 \mathrm{wt} \%, \alpha$ 相 $/ \beta$ 相比为 17.6 , 收率 $3.1 \%$ 。进一步研究表明, 原料中的 微量 $\mathrm{Fe}$ 元素在还原气氛下具有催化作用, 纳米线由 典型的气一液-固(VLS)生长机制控制。

本方法操作简单, 无需催化剂与后续纯化除杂, 产物纯度高、易分离, 可同时获得高品质 $\alpha-\mathrm{Si}_{3} \mathrm{~N}_{4}$ 微 米粉体副产物。

\section{参考文献:}

[1] CHEN L, FENG J. Research status and application of silicon nitride ceramic materials. Journal of Cemented Carbide, 2002, 19(4): 26-229.

[2] XIE T, WU Y C, ZHANG L D. Synthesis and photoluminescence of single-crystalline $\alpha-\mathrm{Si}_{3} \mathrm{~N}_{4}$ nanowires. Journal of Functional Materials, 2004, 35(z1): 3027-3029.

[3] JING G Y, JI H, YANG W Y, et al. Study of the bending modulus of individual silicon nitride nano-belts via atomic force microscopy. Applied Physics A, 2006, 82(3): 475-478.

[4] MAIRE G, VIVIEN L, SATTLE G, et al. High efficiency silicon nitride surface grating couplers. Optics Express, 2008, 16(1): 328-333.

[5] HUANG J, HUANG Z, YI S, et al. Fe-catalyzed growth of one-dimensional $\alpha-\mathrm{Si}_{3} \mathrm{~N}_{4}$ nanostructures and their cathodoluminescence properties. Scientific Reports, 2013, 3: 3504-1-9.

[6] GU Y, LU L, ZHANG H, et al. Nitridation of silicon powders catalyzed by cobalt nanoparticles. Journal of the American Ceramic Society, 2015, 98(6): 1762-1768.

[7] WANG F, HAO Y J, QIN G Q, et al. Effects of the reaction conditions in preparation of $\mathrm{Si}_{3} \mathrm{~N}_{4}$ nanowires. Acta Physico-Chimica Sinica, 2007, 23(10): 1503-1507.

[8] LI K, ZHAO K, WANG Y. In-situ synthesis and growth mechanism of silicon nitride nanowires on carbon fiber fabrics. Ceramics International, 2014, 40(10): 15381-15389.

[9] GUO G F, LI X W, FENG W, et al. Pyrolytic synthesis of singlecrystal $\mathrm{Si}_{3} \mathrm{~N}_{4}$ nanowires by polymeric precursor. Rare Metal Materials and Engineering, 2009, 38(s2): 967-969.
[10] RAN G Z, YOU L P, DAI L, et al. Catalystless synthesis of crystalline $\mathrm{Si}_{3} \mathrm{~N}_{4} /$ amorphous $\mathrm{SiO}_{2}$ nanocables from silicon substrates and $\mathrm{N}_{2}$. Chemical Physics Letters, 2004, 384(1): 94-97.

[11] WANG F, JIN G Q, GUO X Y. Formation mechanism of $\mathrm{Si}_{3} \mathrm{~N}_{4}$ nanowires via carbothermal reduction of carbonaceous silica xerogels. Journal of Physical Chemistry B, 2006, 110(30): 1454614549.

[12] DU H L, ZHANG W, LI Y. Effects of growth parameters on the yield and morphology of $\mathrm{Si}_{3} \mathrm{~N}_{4}$ microcoil prepared by chemical vapor deposition. Materials Research Bulletin, 2014, 50(2): 57-62.

[13] HUANG J, ZHANG S, HUANG Z, et al. Catalyst-assisted synthesis and growth mechanism of ultra-long single crystal $\alpha-\mathrm{Si}_{3} \mathrm{~N}_{4}$ nanobelts with strong violet-blue luminescent properties. CrystEngComm, 2012, 14(21): 7301-7305.

[14] RODRIGUEZ M A, MAKHONIN N S, ESCRINA J A, et al. Single crystal $\beta-\mathrm{Si}_{3} \mathrm{~N}_{4}$ fibers obtained by self-propagating high temperature synthesis. Advanced Materials, 1995, 7(8): 745-747.

[15] CAO Y G, GE C C, ZHOU Z J, et al. Combustion synthesis of $\alpha-\mathrm{Si}_{3} \mathrm{~N}_{4}$ whiskers. Journal of Materials Research, 1999, 14(3): 876-880.

[16] 杨春. 硅粉声场流态化及直接氮化研究. 杭州: 浙江大学硕士 学位论文, 2015.

[17] YUAN C M, LI C, LI G. Kinetic parameters of maize starch in nitrogen atmosphere. Journal of Northeastern University Natural Science, 2012, 33(4): 584-587.

[18] CYPRES R, GHODSI M, LEMPEREUR J P. Direct hydrogenation of carbons by isothermal microthermogravimetry at atmospheric pressure. Fuel Process Technol., 1979, 2: 171-178.

[19] LI Z, GAO W, MENG A, et al. Large-scale synthesis and Raman and photoluminescence properties of single crystalline $\beta$-SiC nanowires periodically wrapped by amorphous $\mathrm{SiO}_{2}$ nanospheres. Journal of Physical Chemistry C, 2009, 113(1): 91-96.

[20] LIU H, HUANG Z, HUANG J, et al. Novel, low-cost solid-liquidsolid process for the synthesis of $\alpha-\mathrm{Si}_{3} \mathrm{~N}_{4}$ nanowires at lower temperatures and their luminescence properties. Scientific Reports, 2015, 5: 17250-1-9.

[21] 唐元洪. 硅纳米线及硅纳米管(精). 北京: 化学工业出版社, 2007: 53-78.

[22] WANG F, JIN G Q, GUO X Y. Sol-Gel synthesis of $\mathrm{Si}_{3} \mathrm{~N}_{4}$ nanowires and nanotubes. Materials Letters, 2006, 60(3): 330-333.

[23] 董文麟. 氮化硅陶瓷. 北京：中国建筑工业出版社，1987: $73-75$. 